\title{
Hellenic Lyceum Cosmic Observatories Network: Status Report and Outreach Activities ${ }^{+}$
}

\author{
Antonios Leisos 1,*(D), Apostolos Tsirigotis ${ }^{1}\left(\mathbb{D}\right.$, George Bourlis ${ }^{1}\left(\mathbb{D}\right.$, Michael Petropoulos ${ }^{1}$, \\ Leonidas Xiros ${ }^{1}$, Ioannis Manthos ${ }^{2}$ (D) and Spyros Tzamarias ${ }^{2}$ \\ 1 Physics Laboratory, School of Science and Technology, Hellenic Open University, 26222 Patras, Greece; \\ tsirigotis@eap.gr (A.T.); bourlis@eap.gr (G.B.); mikepetrop@eap.gr (M.P.); 1xiros@yahoo.com (L.X.) \\ 2 Department of Physics, Aristotle University of Thessaloniki, 54124 Thessaloniki, Greece; \\ ymanthos@gmail.com (I.M.); tzamarias@auth.gr (S.T.) \\ * Correspondence: leisos@eap.gr \\ + This paper is based on the talk at the 7th International Conference on New Frontiers in Physics (ICNFP \\ 2018), Crete, Greece, 4-12 July 2018
}

Received: 30 November 2018; Accepted: 20 December 2018; Published: 22 December 2018

\begin{abstract}
The HELYCON project aims at the installation of cosmic air-shower detectors on the roofs of high-school buildings in western Greece. During the last four years, the HELYCON project made a substantial progress. Three HELYCON stations were installed and are still in operation at the Hellenic Open University (HOU) campus, while a small-scale air-shower detector $(\mu$ Cosmics detector), suitable for in classroom operation, was developed. During the construction and operation of these detectors, many experimental tests and calibration procedures were established, offering the framework for the educational activities of the HELYCON project. In this work, we present the recent developments of the HELYCON project and describe the main aspects of the methodology we use in a five-day training program that introduces the Greek education community to the experimental procedures of HELYCON.
\end{abstract}

Keywords: cosmic rays; air-shower detector; HELYCON; educational cosmic-ray telecopes

\section{Introduction}

In Greek society, and especially within secondary- and higher-education institutions, there is much interest for the contemporary scientific achievements and discoveries of particle physics. However, the involvement of pupils and students with research activities is confined to short visits to large research centers (e.g., Conseil Européen pour la Recherche Nucléaire - CERN) and attendance to demonstration experiments and outreach lectures. Additionally, when student participation in the research process is possible (e.g., masterclasses), this is usually limited to the analysis of preprocessed data, and the experimental process is simplified to a defined algorithmic processing of measurements of physical quantities. On the other hand, there is a recent trend to more actively involve high-school students and teachers to the experimental procedures of particle and astroparticle physics. Toward this direction, many universities and research centers offer wide outreach programs, which are based on the construction, installation, and operation of educational air-shower detectors at high-school buildings.

A typical educational air-shower detector consists of three or four plastic scintillator detectors, connected to photomultiplier tubes (PMT) and read outs by custom-built electronics. The detectors are placed a few meters apart on the roof of a school, college, or university building, registering particles produced in secondary interactions (air shower) after the initial interaction of high-energy cosmic $\left(E>10^{13} \mathrm{eV}\right)$ at the top of Earth's atmosphere. The operations of an educational air-shower detector introduce students to the physics of cosmic rays and to the experimental methodologies and 
procedures of modern high-energy physics experiments. The preparation and assembly of the detector units, as well as the calibration procedures before commissioning, offer the framework for educational activities regarding detector instrumentation, while the operation/monitoring of the detector and data analysis help students understand the main characteristics of cosmic rays and Extensive Air Showers (EAS).

There are many educational cosmic-ray telescopes around the globe [1]. In Greece, the relevant project started at the Hellenic Open University (HOU), the HEllenic LYceum Cosmic Observatories Network (HELYCON) [2]. HELYCON aims to install cosmic air-shower detectors on the roofs of high-school buildings in western Greece, offering the Greek education community the possibility to actively participate in the entire experimental procedure, from the preparation of the detector units to the analysis of the experimental data and the interpretation of the results. By 2014, twelve large $\left(\approx 1 \mathrm{~m}^{2}\right)$ scintillator detectors (HELYCON Detector Modules-HDM) were constructed and tested at the HOU physics laboratory. However, the combined operation of these detectors (i.e., for air-shower detection) was performed inside the lab for limited periods of time, using high-rate digital oscilloscopes. In 2014, three autonomous HELYCON stations were installed at the HOU campus as part of the Astroneu array ${ }^{1}$, thus forming a pilot phase of the HELYCON project [4]. Each station consists of three HDMs arranged in triangular form, and it is equipped with its own data-acquisition system and control/monitor electronics. The HELYCON stations are operated remotely, either independently (single-station operation), or in coincidence with other stations (multiple-station operation) [5].

There are many educational activities that can be performed using an individual HDM and the integrated HELYCON station [6]. Most of these activities (e.g., the calibration of photosensor devices, the estimation of the HDM to Minimum Ionising Particles (MIP), the operation and monitoring of a station, shower reconstruction, and data analysis) can be performed by high-school students under the guidance of their teachers or academic staff. However, due to the large dimensions of the HDM, further studies (e.g., the effect of the geometry of a station) are not applicable to HELYCON stations. For that reason, a small-dimension and portable HELYCON station ( $\mu$ Cosmics detector) was developed [7], suitable for operation inside a high-school classroom. In this work, we present the detector developments and also the utilization of the HDM and the $\mu$ Cosmics detector in the framework of a five-day training program for high-school students. In Section 2 we briefly describe the HELYCON station, while in Section 3 we outline the architecture of the $\mu$ Cosmics detector. Section 4 focuses on the activities of the training program, while in Section 5 the conclusions are drawn.

\section{HELYCON Station}

Each HELYCON station consists of three HDMs forming a triangle with a typical separation of about 20-30 m between them (see Figure 1). Each HDM is a $1 \mathrm{~m}^{2}$ plastic scintillator detector made of two $80(12 \times 10 \times 0.5) \mathrm{cm}^{3}$ layers of scintillator tiles per layer, wrapped in reflective paper (Dupont Tyvek 2460B, Dupont, Wilmington, DE, USA) and placed in position in a wooden frame. The scintillation material is IHEP SC-301 containing $2 \%$ pTP (p-terphenyl) and $0.02 \%$ POPOP (1,4-bis(5-phenyloxazol-2-yl) benzene) dopants. The generated scintillation light, when a particle activates the detector, is collected by wavelength-shifting (WLS) optical fibers (Bicron BCF91-A, Bicron Corporation, Newbury, OH, USA) that are embedded inside grooves built into the scintillator tiles, and it is driven to a fast Photonis XP1912 photomultiplier tube (HZC photonics, Hainan, China) for detection. The assembled detector is shielded against ambient electronic noise by a thick 0.4 $\mathrm{mm}$ aluminum foil that is wrapped around the wooden frame. Finally, the wrapped detector is placed inside a water-proof wooden container, made out of boat-building wood, which is consequently covered with a reflective insulator (Tyvek, Wilmington, DE, USA), to prevent overheating and excessive

1 The Astroneu array is a hybrid air-shower detector array consisting of 12 charge particle detectors (HDMs) and six radio frequency antennas that detect RF signals emitted by very high energy $\left(E>10^{17} \mathrm{eV}\right)$ showers [3-5]. 
humidity inside the detector. By requiring three or more of these detectors to register particles within a narrow time window, one can distinguish between the relatively high flux of individual particles from low-energy cosmics and the lower rate of extensive air showers caused by higher-energy primaries. Measuring the relative times that the shower front passes through the particle detectors and using triangulation, the direction of the shower axis and, consequently, of the primary particle can be reconstructed with an accuracy of a few degrees. Furthermore, using the global positioning system (GPS) to provide an absolute time reference, data from distant stations can be combined.

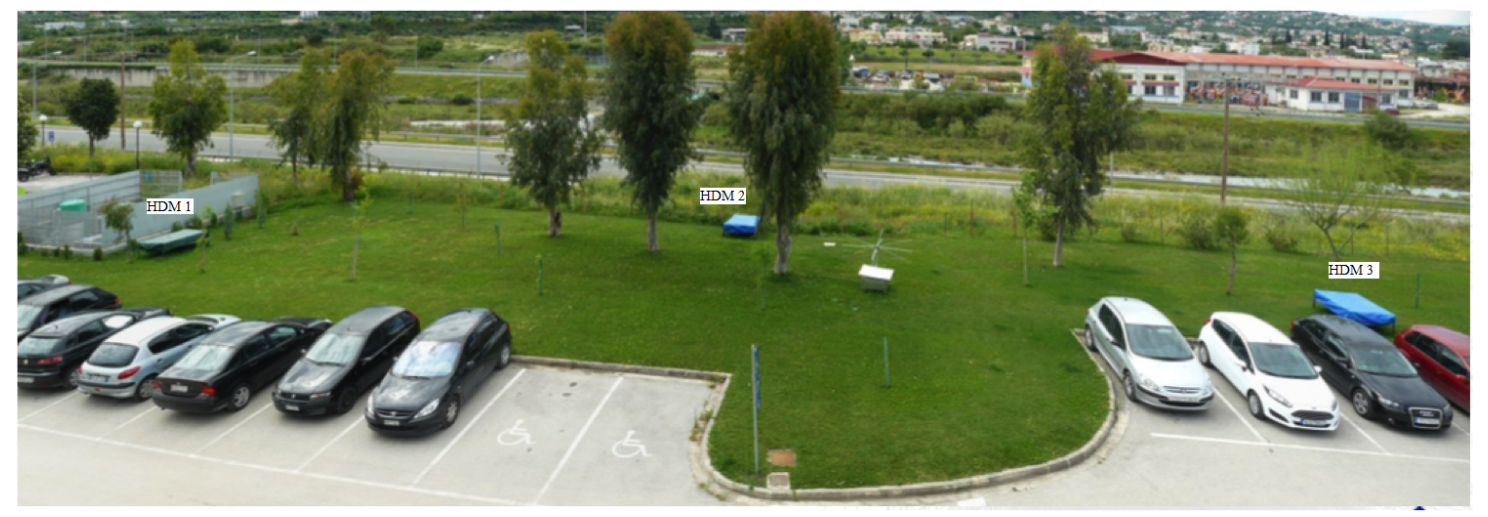

Figure 1. HELYCON station installed at the Hellenic Open University (HOU) campus. Three denoted boxes are the HELYCON Detector Modules (HDMs) of Station B.

The Data Acquisition (DAQ) system of the HELYCON station is based on the Quarknet DAQ board [8]. The Quarknet board registers the times of the crossings of the waveforms with an adjustable threshold, allowing the timing of the pulses and the estimation of their size using the Time over Threshold value (ToT). A local computer (Station Local Computer-SLC) is used for the control and monitor of the station, and data acquisition, as well as for communication with the physics laboratory's main server. The Quarknet board is managed through specific software, developed at Fermilab in LabVIEW, which runs on the SLC. Apart from managing the data transfer to the SLC hard disk, it provides the means to set the operating parameters of the board. These are related to the input channels' activation, the amplitude threshold values for each active channel, the trigger majority level, the trigger window width, the maximum time duration of an event, as well as to actions such as start/stop acquisition, monitoring the acquisition progress and event rates, and recording measurements from the environmental sensors.

The operating parameters of the HDMs are managed by online software packages (HELYCON control software) that provide a graphical user interface to select the operating voltage and the corresponding ramp-up time for each of the detectors' PMT. In parallel, it continuously monitors and logs the PMTs' actual voltages and the detectors' temperature. The HELYCON control software displays, in real time, the current values and history plots of the operating voltages and temperatures, also providing alarms and taking emergency actions (e.g., if selected by the user, it shuts down or lowers the voltages) in case the operating voltages or the detector temperature exceed predefined limits. In addition, the control and monitoring software decodes the Quarknet data and performs a fast reconstruction of the shower axis direction in real time. The timing information of the acquired pulses (timestamp and Time over Threshold) as well as a 3D visualization of the shower event are displayed on a web browser, while the decoded data are also used for online data-quality monitoring.

The simulation software is part of the Hellenic Open University Simulation and Reconstruction software (HOURS) $[9,10]$. The software describes in detail the detector components and is interfaced with the COsmic Ray SImulations for KAscade (CORSIKA) package [11], which simulates the development of the EAS and provides particle information at the detector level. The HOURS software uses parameterizations for the scintillation process and the effect of the WLS fibers, which are determined either by detailed simulation using the GEometry ANd Tracking (GEANT4) package [12] 
or by using the results of the calibration procedure [4]. The photons reaching the PMT are used to generate the photoelectrons and the PMT response pulse. Finally, signal transmission (cable effects) and the digitization electronics functionality are simulated to produce the event sample with the same format as the raw data.

The performance of each HELYCON station has been evaluated in detail [5] and found to be in excellent agreement with Monte Carlo (MC) predictions.A typical HELYCON station reconstructs extensive air showers with an energy threshold of about $10 \mathrm{TeV}$ and at a rate of about 17 events per hour. The angular resolution of the station is about 3.5 degrees, while the zenith and the azimuth direction of the shower axis is estimated with an accuracy of 3 and 10 degrees, respectively [5].

\section{3. $\mu$ Cosmics Detector}

Disadvantages of the HELYCON station are that it is massive, hard to move, and quite expensive. In addition, the roof of a school is not always available for deploying detectors. To overcome this situation, the Physics Laboratory of HOU constructed a small-scale HELYCON station with detector units occupying $1 / 6$ of the area of the HELYCON detector module. This portable air-shower detector ( $\mu$ Cosmics detector) can be operated inside the classroom or the high-school lab, is very easy to carry and much cheaper than the HELYCON station, and free of high-voltage circuits. In addition, the $\mu$ Cosmics detector can easily be constructed by the students, while the small dimension and weight of the units offer more activities not foreseen with HELYCON stations, i.e., coincidence studies and geometries studies. On the other hand, the relatively small interdetector distance and the small active area of the $\mu$ Cosmics detector results in poorer angular resolution, as well as to a lower acquisition rate in comparison to a HELYCON station. However, there is no restriction on the associative physics studies that can be performed (e.g., the angular distribution of EAS) with the $\mu$ Cosmics detector.

The architecture of the $\mu$ Cosmics detector [7] follows the architecture of the HELYCON station (Figure 2). The active area of the detector unit is $36 \times 40 \mathrm{~cm}^{2}$, made up of two layers of scintillation tiles (12 tiles per layer). The WLS fibers (36 per detector unit) are used to guide the light to a Silicon PhotoMultiplier (SiPM). The scintillator is wrapped with reflective paper (Tyvek), and the fibers at one end are glued in a plastic rectangular head, while the other end is covered with reflective paint. The Silicon Photomultiplier (SiPM) (PM6650-EB, KETEK, Munich, Germany) is made of 12,272 cells (50 $\mu \mathrm{m}$ cell size) and is connected to the fibers end using a rectangular plastic interface. The SiPM is operated at 30-40 Volts, and power is supplied by a DC-DC converter (HMA-0.2N2.5-5, highvolt.de, Hamburg, Germany) which is controlled by a USB module (LucidControl AO4, Lucidcontrol, Kaufbeuren, Germany). The scintillators and the SiPM, along with the power electronics, are attached to a wooden frame that is inserted inside a box made of PVC. The overall dimension of the detector unit is $67 \times 42 \times 7 \mathrm{~cm}^{3}$ and weighs $6 \mathrm{~kg}$.
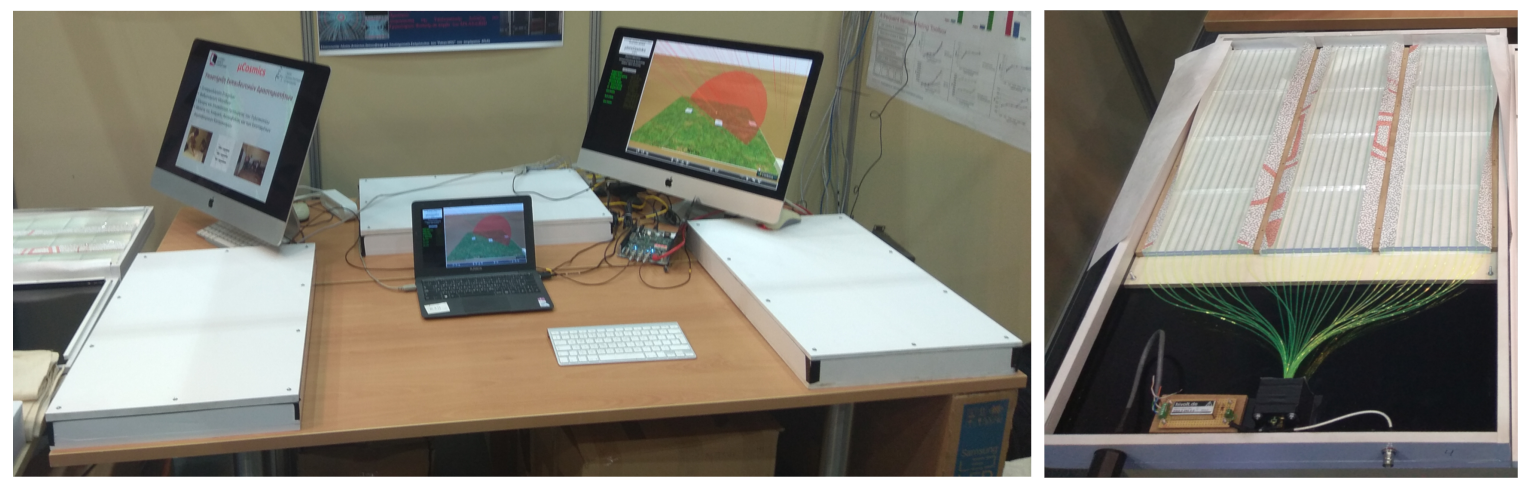

Figure 2. (left) $\mu$ Cosmics detector with three detector units and a Quarknet card; (right) internal structure of the detector unit. 
For the data-acquisition system, the Quarknet card could be used; another option is a commercial PC-based oscilloscope that offers full waveform information but lacks trigger logic and GPS time-stamping. Monte Carlo studies show that the resolution of a detector with four units arranged in a square sized $10 \mathrm{~m}$ is about 6.5 degrees, while the rate of reconstructed showers is 10 per hour, which is is suitable for the duration of a lesson ( $45 \mathrm{~min}$ ) in a Greek lyceum.

\section{Five-Day Educational Program Using the HELYCON Station and the $\mu$ Cosmics Detector}

A five-day educational program was designed by the physics laboratory of HOU in order to engage high-school students and teachers with the experimental methodology of constructing and operating an air-shower detector. For that purpose, four tasks were prepared to be carried out by students: (a) construction of a $\mu$ Cosmics detector unit (Task A), (b) calibration of a PMT (Task B), (c) response of HDM to MIP (Task C), and (d) operation of a HELYCON station and collection of shower data (Task D).The first three tasks describe the main aspects of an air-shower detector. The construction of the $\mu$ Cosmics detector highlights the physical processes that are taking place inside the detector units, PMT calibration describes the relation between physical quantities and measurable electric signals, while the response of the HDM to MIPs outlines the operation of the integrated detector. Finally, the results of these tasks offer the necessary information in order to set the operational parameters of a HELYCON station and to collect shower data (Task D).

The construction of the $\mu$ Cosmics detector unit includes the assembly of the unit's components (scintillator tiles, WLS fibers, SiPM, and electronic devices). The wooden frame and the outer plastic box are prepared in advance having the necessary holes for the attachment of the components. Students do not use any dangerous equipment or high-voltage electrical circuits. During the first two days, students prepare the scintillator tiles, the Tyvek reflective paper, and the WLS fibers, as well as the cable connectors to the plastic box (Figure 3). On the last day of the task, they check the operation of the detector unit. First, they ensure that the detector unit is light-proof by measuring the current drawn by the SiPM when it is illuminated with a flashlight. Then, the unit is tested using cosmics. The detector unit is placed between two already completed and calibrated detector units, and signals are acquired by a high-rate digital oscilloscope. The outer detectors are used to trigger the oscilloscope in coincidence while the pulses of the under test detector are examined. Special software evaluates pulse-height distribution, which is compared with the corresponding distributions of well-working detectors.

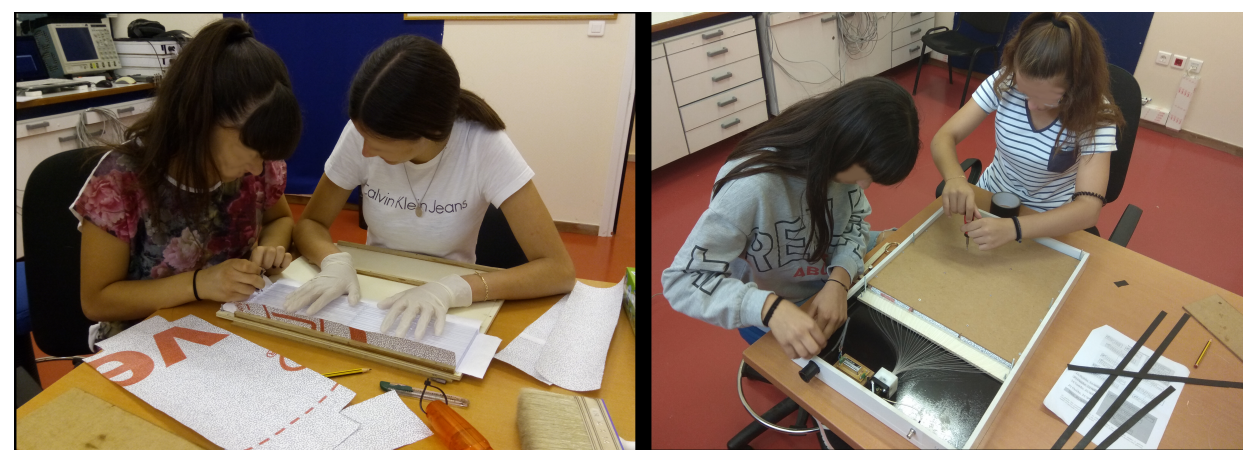

Figure 3. Students working on the construction of the $\mu$ Cosmics detector unit. (Left) tiles are placed inside the wooden frame and wrapped with reflective paper. (Right) students attach the wooden frame inside the plastic box.

The calibration of the HELYCON photosensors includes the determination of the PMT gain and the mean pulse height and charge distributions of the PMT waveforms when practically only one phototelectron is emitted from the photocathode (single-photoelectron conditions). The experimental setup of this activity is shown in Figure 4 (left). A pulse generator is used to drive an LED that illuminates the PMT, both installed inside a lightproof box. The PMT waveforms are acquired by a digital oscilloscope that is triggered by the pulser. The digital oscilloscope is managed through specific 
software developed for this experiment, hiding the details of the electronic units from students who only change the light intensity of the source and the high voltage of the PMT. The software acquires pulses and evaluates the charge (pulse integration) and peak voltage of each pulse, producing the relevant histograms that appear on the screen (Figure 4, right). In this activity, special attention is given to the stochastic properties of the peak amplitude and the charge of the pulses since no processing is applied, i.e., the distributions of the peak amplitude and charge of the pulses are not fitted by any modelling functions. The only information that is used is the position of the peak of the distributions in order to explore the relation of the peak position with the intensity of the light ${ }^{2}$.
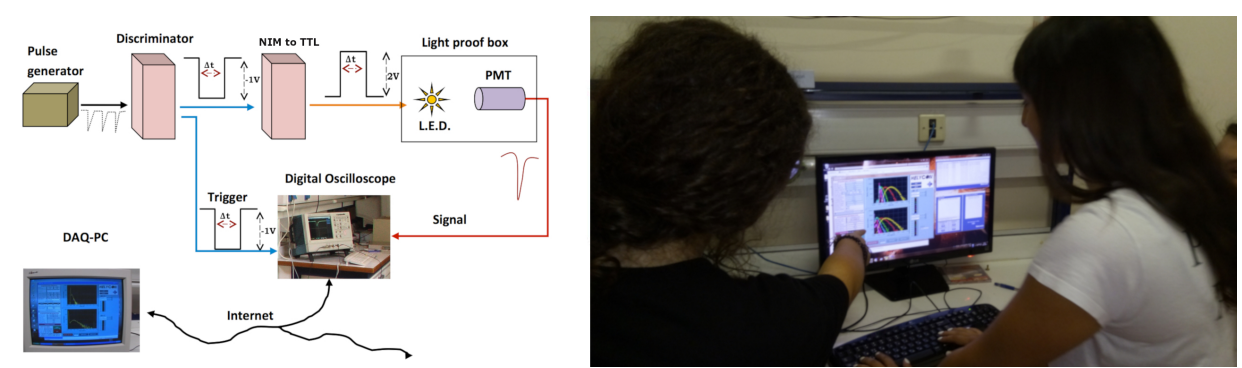

Figure 4. (Left) A sketch of the experimental setup for the PMT calibration task. (Right) Students examining the histograms presented by the data-acquisition software.

In the third task, students quantify the response of an HDM to MIPs exploring also the effect of the distance between the incident position of the MIP and the PMT position to the signal strength. A small hodoscope (two small scintillator counters with an absorber in between) is used to select muons that hit one HDM (Figure 5, left). The coincidence of the two signals of the hodoscope is used to trigger a digital oscilloscope that acquires the HDM waveforms. Special software, developed for this application, is used to acquire the pulses of the HDM PMT and to evaluate the peak voltage and the charge of the waveform presented as histograms after the accumulation of many events (Figure 5, right). In this activity the students take measurements for 9 different incident positions (regions) of the MIP on the HDM. The regions are predefined and marked on the aluminum foil of the HDM. Then they produce the HDM homogeneity map i.e., the relative signal strength on these 9 regions and explore the relation of the MIP incident position with the response of the HDM predicting the result when moving the hodoscope closer or away from the HDM PMT.
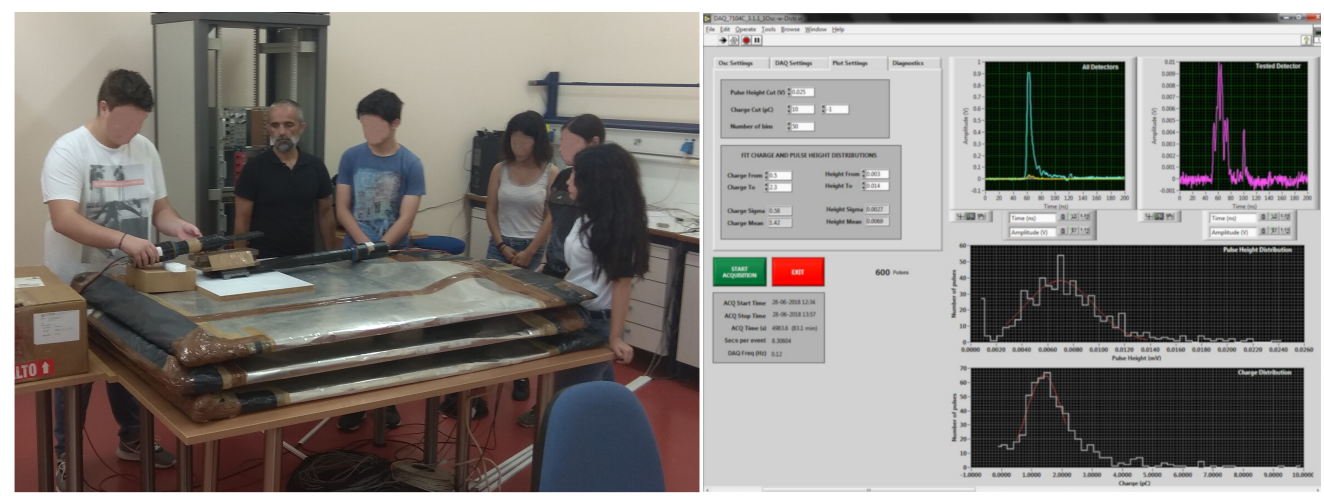

Figure 5. (Left) students placing the hodoscope above the HDM. (Right) screenshot of the data-acquisition software used by the students.

2 As intensity goes higher, the x-position of the peak also moves to higher values but, on the contrary, when intensity is sufficiently low, the peak remains in the same x-position, while the y-position of the peak becomes lower (single-photoelectron condition). 
The results of these tasks are used to set the operational parameters of the PMTs of a HELYCON station. For example, using the gain of the PMT (Task B) and the response of the HDM to MIPs (Task C) as a reference, the students evaluate the operating High Voltage (HV) of each HDM of the HELYCON stations in order for all the HDMs to have the same response.During this activity (Task D), students collect shower data, watch the 3D representation of the showers and confirm that showers from above are more frequent than very inclined showers. They also estimate the rate of recorded showers under different operation conditions, i.e., different threshold values and/or coincidence level.

The HELYCON five-day educational program was implemented in 2018 at the physics laboratory of HOU. Fifteen pupils (The pupils had just completed the first grade of lyceum) from the area of Patras, worked for a week at the HOU physics laboratory in the framework of a summer school in astroparticle physics. Half of the students were selected according to their overall grade, while half of them by lot. The students were arranged in three groups, each responsible for Tasks A to C. The first day was devoted to lectures, while the following three days were devoted to the completion of the tasks. In each of these three days, the students worked for at least $4 \mathrm{~h}$ in the lab, while on the last (fifth) day, the three groups gathered in the HELYCON control room, where they operated the three stations of the Astroneu array collecting air showers (Task D). The trainer team was chosen to comprise academic staff but also high-school teachers that had experience with HELYCON operations and procedures. The tutors of the tasks had prepared educational material suitable for the high-school students, along with questionnaires that were filled by the students at the end of each day. The three groups were in close collaboration with each other, communicating in every-day meetings the methodologies and the problems they faced during their work. This way, each group was aware of the progress of the other groups, realizing that, although independent, the three tasks were equally important in conjunction, and necessary for the main objective: to construct and operate an air-shower detector.

The tasks were presented to the students, leaving them to freely choose in which task they would like to participate. The most popular was the construction of the $\mu$ Cosmics detector unit. Although this activity does not make use of any modern instruments or computers, the manual labor using simple tools (scissors, knives, screwdrivers, etc.) attracted more students to this task. On the other hand, the students of the other two activities (Tasks B and C) had to deal with modern equipment and integrated detectors. However, after the first day in the lab, they familiarized themselves with the experimental setup and soon felt confident to use the instruments and the software. They realized that physical processes, invisible to the eye, can still be examined by the electric signals they produce and that they could predict the effects by changing the experimental setup. They also realized that experimental and statistical errors affect the measurements, and that experimental failures are always expected (For example, during school, the monitoring link of one HDM was broken).

The time schedule of the educational program was found to be sufficient for the completion of Tasks A, B, and C. However, the dedicated time (one day) for station operation (Task D) was not enough. The students did not calculate the shower direction by themselves even though how to do it was explained. It was also evident that, among the tutors, the high-school teachers had the necessary experience of modifying the pedagogical approach according to the pupils' level of knowledge, establishing better communication with them. However, these two drawbacks could be diminished if the tutor team consists purely of high-school teachers that could also give the first-day lectures within the classroom and dedicate another day to Task D.

\section{Discussion}

In 2014, three autonomous HELYCON stations were installed at the HOU campus, fully equipped with control and digitization electronics, as well as a GPS system for absolute time stamping. Offline and online software was developed and extensively tested, while calibration procedures for the detector components, the DAQ system, as well as for the integrated station were advanced. The stations are operated remotely, and the performance of each station, as well as of the whole array, has been studied in detail. In addition, a portable and low-cost HELYCON station, suitable for in-classroom operation, 
has also been developed. The $\mu$ Cosmics detector expands the educational activities of the HELYCON project, also offering the possibility to operate as a muon telescope. It can be operated autonomously using a PC-based digital oscilloscope or in coincidence with distant detectors using the Quarknet card.

After the successful installation and operation of the three HELYCON stations and the construction of the $\mu$ Cosmics detector, the next step for the HELYCON project was the utilization of these detectors for the education of high-school students. A five-day training program was designed to actively engage high-school students to the main aspects of an air-shower detector. In this first attempt, the comments we received from the students were very positive and the overall picture was very encouraging. The physics of cosmic rays and the concept of multimessenger astronomy fascinated the students. A common misconception among all students that telescopes are instruments with a lens observing the visible light of distant objects was cleared. It is also very interesting that many students had selected subjects (for the following school year) from the Humanities orientation group instead of the Sciences orientation group. However, since in the first grade of lyceum the subjects of the curriculum are common to all students and all of them were very enthusiastic for their task, we did not notice any difference on the performance of each orientation group. On the other hand, pupils with high grades were more confident and more active during their work.

The impact of this educational program to the Greek education community was very appreciated and many schools have been scheduled to attend it in the following year. The feedback of a larger sample of pupils will allow us to evaluate the methodologies we use and take proper actions. Furthermore, the application of such training programs by science teachers at high-school laboratories using their own $\mu$ Cosmics detector or HELYCON station is foreseen for the near future. At the time of writing, two $\mu$ Cosmics detectors are about to be used by high-school teachers for a pilot operation within the classroom, while one HELYCON station has been granted for remote operation, exploiting the use of its infrastructure as a telelaboratory. Finally, educational material was prepared and new student activities were designed in order for the HELYCON project to reach a level of maturity to receive funding toward a wide outreach program for the physics of cosmic rays in Greece.

Author Contributions: Conceptualization, A.L. and S.T.; funding acquisition, A.L.; methodology, A.L., A.T., G.B., M.P., L.X., I.M., and S.T.; project administration, A.L. and S.T.; software, A.T. and G.B.; validation, A.T., G.B., M.P., and L.X.; writing-original draft, A.L.

Funding: This research was funded by the Hellenic Open University Grant No. $\Phi K$ 228: "Development of technological applications and experimental methods in Particle and Astroparticle Physics".

Conflicts of Interest: The authors declare no conflict of interest.

\section{References}

1. See for Example: International Cosmic Day. Available online: https://icd.desy.de/e49245/ (accessed on 30 November 2018).

2. HELYCON. Available online: helycon.eap.gr (accessed on 30 November 2018).

3. Manthos, I.; Gkialas, I.; Bourlis, G.; Leisos, A.; Papaikonomou, A.; Tsirigotis, A.G.; Tzamarias, S.E. Cosmic Ray RF detection with the ASTRONEU array. arXiv 2017, arXiv:1702.05794.

4. Avgitas, T.; Bourlis, G.; Fanourakis, G.K.; Gkialas, I.; Leisos, A.; Manthos, I.; Tsirigotis, A.; Tzamarias, S.E. Deployment and calibration procedures for accurate timing and directional reconstruction of EAS particle-fronts with Helycon stations. arXiv 2017, arXiv:1702.04902.

5. Avgitas, T.; Bourlis, G.; Fanourakis, G.K.; Gkialas, I.; Leisos, A.; Manthos, I.; Stamelakis, A.; Tsirigotis, A.; Tzamarias, S.E. Operation and performance of a pilot HELYCON cosmic ray telescope with 3 stations. arXiv 2018, arXiv:1801.04768.

6. Leisos, A.; Avgitas, T.; Bourlis, G.; Fanourakis, G.K.; Gkialas, I.; Manthos, I.; Stamelakis, A.; Tsirigotis, A.G.; Tzamarias, S.E. The Hellenic Open University Cosmic Ray Telescope: Research and Educational Activities. EPJ Web Conf. 2018, 182. [CrossRef]

7. Tsirigotis, A.G.; Leisos, A. $\mu$ Cosmics: A low cost educational cosmic ray telescope. In Proceedings of the ICNFP 2018, Crete, Greece, 4-12 July 2018. 
8. Hansen, S.; Jordan, T.; Kiper, T.; Claes, D.; Snow, G.; Berns, H.; Burnett, T.H.; Gran, R.; Wilkes, R.J. Low-cost data acquisition card for school-network cosmic ray detectors. IEEE Trans. Nucl. Sci. 2004, 51, 926-930. [CrossRef]

9. Tsirigotis, A.G.; Leisos, A.; Tzamarias, S.E. HOU Reconstruction \& Simulation (HOURS): A complete simulation and reconstruction package for very large volume underwater neutrino telescopes. Nucl. Instr. Meth. A 2011, 626, 185-187.

10. Tsirigotis, A.G.; Leisos, A.; Tzamarias, S.E. Reconstruction efficiency and discovery potential of a Mediterranean neutrino telescope: A simulation study using the Hellenic Open University Reconstruction and Simulation (HOURS) package. Nucl. Instr. Meth. A 2013, 725, 68-71. [CrossRef]

11. Heck, D.; Knapp, J.; Capdevielle, J.N.; Schatz, G.; Thouw, T. CORSIKA: A Monte Carlo Code to Simulate Extensive Air Showers. Forschungszentrum Karlsruhe Report FZKA 6019 (1998). Available online: https:/ / www.ikp.kit.edu/corsika/70.php (accessed on 30 November 2018).

12. GEANT4 Collaboration. Geant4-A simulation toolkit. Nucl. Instrum. Methods Phys. Res. A 2003, 506, 250-303. [CrossRef]

(c) 2018 by the authors. Licensee MDPI, Basel, Switzerland. This article is an open access article distributed under the terms and conditions of the Creative Commons Attribution (CC BY) license (http:/ / creativecommons.org/licenses/by/4.0/). 